\title{
SOME REMARKS ON TIME, UNCERTAINTY, AND SPIN
}

\author{
Robert Carroll \\ University of Illinois \\ email: rcarroll@math.uiuc.edu
}

March, 1999

\begin{abstract}
Some observations are made about energy-time uncertainty and spin in the context of trajectories as in Faraggi-Matone or Floyd.
\end{abstract}

\section{INTRODUCTION}

The background here is properly the Bohmian or trajectory approach to quantum mechanics (QM) (cf. [3, 22] for technical details and [2, 5, 21, 25] for perspectives, philosophy, etc.). In a seminal paper [10] Faraggi and Matone (FM) develop a 1-D trajectory theory based on a deep equivalence principle which seems to provide the proper foundational structure for such theories (cf. also [11, 12]). For example quantization is a direct consequence of the equivalence principle and there is a nontrivial action even for bound states. In particular in 10] one avoids a flaw in the Bohm theory based on the erroneous assumption that particle velocity $\dot{q}$ is the same as $p / m=\partial_{q} S_{0}=S_{0}^{\prime}$ where $S_{0}=W$ is Hamilton's characteristic function or reduced action $\left(S=S_{0}-E t\right)$. The correct version here is $p=\partial_{q} W=m \partial_{\tau} q$ where $\tau-\tau_{0}=m \int_{q_{0}}^{q}\left(d x / \partial_{x} W\right)$ represents a time concept developed by Floyd (cf. 15, 16, 17, 18, 19, 20]) in studies of trajectory representations and microstates. In particular one can work with $t \sim \partial_{E} W$ to write $t-t_{0}=\partial_{E} \int_{q_{0}}^{q} W^{\prime} d x$ and arrive at $m \dot{q}=m(d t / d q)^{-1}=$ $m / W_{E}^{\prime}=W^{\prime} /\left(1-\partial_{E} Q\right)$ where $Q$ is the quantum potential $Q=\left(\hbar^{2} / 4 m\right)\{W, q\}$ (Schwartzian derivative - details below). Given even alone this important variation from the traditional Bohm theory (and more generally, given [10]) many philosophical discussions (some quite recent) regarding trajectory representations should be drastically modified. We will mostly avoid philosophy in this note and simply make a few comments about matters related to this use of time. One notes in [10] also that a formula $p=m_{Q} \dot{q}$ can be obtained via the use of a quantum mass $m_{Q}=m\left(1-\partial_{E} Q\right)$. The quantum potential $Q$ is regarded here as the particles reaction to an external potential $V$ and no pilot-wave philosophy is needed. We will consider stationary states but allow $E$ to vary continuously (so discrete eigenvalues $E_{n}$ are not indicated although some arguments could be adjusted to include them). 


\section{BACKGROUND}

We sketch briefly some background. First one starts with $i \hbar \psi_{t}=-\left(\hbar^{2} / 2 m\right) \psi_{q q}+V \psi$ for stationary states $\psi=\psi(q) \exp (-i E t / \hbar)$ so $i \hbar \psi_{t}=E \psi$ and $-\left(\hbar^{2} / 2 m\right) \psi^{\prime \prime}+V \psi=E \psi$ (' $\left.\sim \partial_{q}\right)$. "Classically" one takes then $\psi=\operatorname{Rexp}(i W / \hbar)$ where $S=S_{0}-E t$ and $S_{0}=W$ to arrive at

$$
\frac{1}{2 m}\left(W^{\prime}\right)^{2}+V+Q-E=0 ;\left(R^{2} W^{\prime}\right)^{\prime}=0
$$

where $(\bullet) Q=-\hbar^{2} R^{\prime \prime} / 2 m R$ is the quantum potential. In [10] this whole procedure is changed and the matter is developed in the context of a general equivalence principle leading to a quantum stationary Hamilton-Jacobi equation (QSHJE)

$$
\frac{1}{2 m}\left(W^{\prime}\right)^{2}+\mathcal{W}(q)+Q(q)=0
$$

which is actually an identity. The individual terms are (here $\{f, q\}=\left(f^{\prime \prime \prime} / f^{\prime}\right)-(3 / 2)\left(f^{\prime \prime} / f^{\prime}\right)^{2}$ is the Schwartzian derivative)

$$
\mathcal{W}(q)=-\frac{\hbar^{2}}{4 m}\left\{\exp \left(\frac{2 i W}{\hbar}\right), q\right\} ; Q(q)=\frac{\hbar^{2}}{4 m}\{W, q\}
$$

This $Q$ is the general quantum potential which in the special context of (2.1) is $Q=$ $-\left(\hbar^{2} / 2 m\right)\left(R^{\prime \prime} / R\right)$ as in $(\bullet)$ (note $R^{2} W^{\prime}=c$ from (2.1) will produce the Schwartzian derivative $\{W, q\}$ as in (2.3)). Further one can identify $(\bullet \bullet) \mathcal{W}(q)=V-E$ for which we refer to [10]. Thus given $V$ one can determine $W$ via $(\bullet \bullet)$ or via (2.2) with $\mathcal{W}=V-E$. Note that (2.2) is a third order differential equation for $W$ and its solutions will lead to microstates à la Floyd.

Now in Floyd's work [15] it was apparently first observed that Bohm's assumption $p=W^{\prime}=m \dot{q}$ (for particle velocity $\dot{q}$ ) is not generally valid and the correct version is (cf. [10])

$$
\begin{gathered}
m\left(1-\partial_{E} Q\right) \dot{q}=W^{\prime} \equiv m_{Q} \dot{q}=W^{\prime} \equiv m \partial_{\tau} q=W^{\prime} \\
m_{Q}=m\left(1-\partial_{E} Q\right) ; \tau-\tau_{0}=m \int_{q_{0}}^{q} \frac{d x}{W^{\prime}}
\end{gathered}
$$

Then one has, using (2.1) and Floyd's effective or modified potential $U=V+Q$,

$$
t-t_{0}=\partial_{E} \int_{q_{0}}^{q} W^{\prime} d x=\left(\frac{m}{2}\right)^{1 / 2} \int_{q_{0}}^{q} \frac{\left(1-\partial_{E} Q\right) d x}{\sqrt{E-U}}
$$

and $d \tau / d t=1 /\left(1-\partial_{E} Q\right)$. Thus $t$ is explicitly a function of $E$ and we want to expand upon this aspect of the theory. It is important to note that general solutions of the Schrödinger equation above should be taken in the form $(\mathbf{2 A}) \psi=\left(W^{\prime}\right)^{-1}[A \exp (-i W / \hbar)+B \exp (i W / \hbar)]$ and $p \sim \partial_{q} W=W^{\prime}$ is the generic form for $p$ corresponding to momentum in a quantum mechanical Hamiltonian $(1 / 2 m) p^{2}+V \sim(1 / 2 m)\left(-i \hbar \partial_{q}\right)^{2}+V$. Thus $p=W^{\prime}$ corresponds to $p \sim-i \hbar \partial_{q}$ and this is the quantum mechanical meaning for $p$; it will not correspond in general to mechanical momentum $m \dot{q}$ for particle motion. 


\section{GENERAL COMMENTS IN 1-D}

First one sees that Hamilton-Jacobi (HJ) procedures involve $t \sim \partial_{E} W$ and we will modify an argument in [13] in order to give further insight into the relation $t=t(E)$. We think of a general stationary state situation with $S \sim W-E t=W(q, E)-E t$ so that $\partial S / \partial t=-E$ and $t=t(E)$. Setting $\mathcal{S}=-S$ with $\partial \mathcal{S} / \partial t=E$ we can write then $(\mathbf{\beta}) W=E t-\mathcal{S}=t \mathcal{S}_{t}-\mathcal{S}$ in Legendre form. Now given $W=W(E, q)$, with $q$ fixed, one has $\partial_{E} W=t+E t_{W}-\mathcal{S}_{t} t_{W}=t$ so $(\boldsymbol{\beta}) \mathcal{S}=E W_{E}-W$ gives the dual Legendre relation. Consequently the constructions in 10] for example automatically entail the Legendre transformation relations ( $)$ and (\$) involving $\mathcal{S}=-S$ and $W$.

Now one comes to the energy-time uncertainty "principle" which should be mentioned because of situations involving energy dependent time for example (cf. [1, 4, 6, 7, 14, 30] for various approaches - we make no attempt to be complete or exhaustive here). First, in a perhaps simple minded spirit, let us recall that microstates are compatible with the Schrödinger representation by wave functions $\psi$ and hence one will automatically have a connection of the trajectory representation with Hilbert space ideas of observables and probability (more on this below). In the Hilbert space context the uncertainty $\Delta q \Delta p \geq \hbar / 2$ is a trivial consequence of operator inequalities and we take it to mean nothing more nor less ( $\Delta p$ for example represents a standard deviation $<\psi\left|(\hat{p}-<\hat{p}>)^{2}\right| \psi>$ where $\hat{p} \sim-i \hbar \partial_{q}$ ). In this spirit nothing need be said about measurement and we will not broach the subject in any way except to say that sometimes for a trajectory we will think e.g. of physical increments $\delta q \sim q-q_{0}$ and $\delta p \sim p-p_{0}$. Thus we will try to maintain a distinction between $\delta q$ and $\Delta q$ for example and we do not require that $\delta q$ be measured, only that it be a natural mathematical concept. After all $\Delta q$ above is also only a natural mathematical concept without any a priori connection to measurement. The idea of attaching physical meaning to $\Delta q$ via measurement seems no stranger than thinking of $\delta q$ as a meaningful possibly measurable physical quantity. As for energy-time uncertainty we remark first that if one departs by $\epsilon$ from a correspondence between observables and self-adjoint operators then an $\epsilon$ approximate inequality (ET) $\Delta E \Delta t \geq \hbar / 2$ can be proved in a Hilbert space context (see e.g. [14 for a detailed discussion). There are also various crude physical derivations based on $\delta q \sim(p / m) \delta t$ where $p$ is the physical momentum and subsequently, for $\delta E \sim(p / m) \delta p$ when e.g. $E \sim\left(p^{2} / 2\right)+V(q)$, one often writes $($ EET $) \delta p \delta q \sim(p / m)(m / p) \delta E \delta t=\delta E \delta t \geq \hbar / 2$ based on a $q-p$ uncertainty with $\delta q \sim \Delta q$ etc. (displacement version). This would be fine if $p=m \dot{q}$ but we have seen that $p$ has an unambiguous quantum mechanical meaning as in Section 2 and the argument involving $\delta q=(p / m) \delta t$ is generally not valid. A more convincing argument can be made via use of Ehrenfest type relations $(\hat{H} \sim E)$

$$
\frac{d<\hat{Q}>}{d t}=\frac{1}{\hbar}<i[\hat{H}, \hat{Q}]>; \delta E \delta Q \geq \frac{\hbar}{2}\left|\frac{d}{d t}<\hat{Q}>\right|
$$

and an argument that the time $\delta t$ for a change $\delta Q=\delta<\hat{Q}>$ should be $\delta t=\delta Q / \mid d<$ $\hat{Q}>/ d t \mid$, leading to $(\mathcal{E} \mathcal{E} \mathcal{T}) \delta E \delta t \geq \hbar / 2$ (without intervention of $p$, where however one has $d<q>/ d t \sim(1 / m)<p>)$. 
Since the beginning step $\delta q \sim(p / m) \delta t$ is generally wrong in the crude argument above let us adjust this following (2.4) to be $\delta q \sim(p / m) \delta \tau \sim\left(p / m_{Q}\right) \delta t$ where $p=W^{\prime}$ is the conjugate momentum (QM momentum). Then with $\delta E \sim(p / m) \delta p$ one will arrive at $\delta p \delta q \sim \delta E \delta \tau \sim \delta E \delta t /\left(\left(1-Q_{E}\right)\right.$ and consequently a correct displacement (or perhaps trajectory) version of (ET) should be

$$
\delta E \delta \tau \geq \frac{\hbar}{2} \equiv \delta E \delta t \geq\left(1-\partial_{E} Q\right) \frac{\hbar}{2}
$$

Since in the trajectory picture we are dealing with $t=t(E)$ via $t=\partial_{E} W$ (with $E$ a continuous variable here) one will have $\delta t=W_{E E} \delta E$ so (3.2) requires a curious condition $($ \$क) $)(\delta E)^{2} \geq\left[\left(1-Q_{E}\right) \hbar / 2 W_{E E}\right]$. Thus apparently for any energy change compatible with the microstate picture (\$op) must hold. This would seem to preclude any positive infinitesimal $\delta E$ unless $\left(1-Q_{E}\right) / W_{E E} \leq 0$ (with restrictions on any negative $\delta E$ ). One can perhaps envision here microstates as developed by Floyd generated at energy $E_{0}$ with initial conditions $W_{0}\left(E_{0}\right), W_{0}^{\prime}\left(E_{0}\right)$, and $W_{0}^{\prime \prime}\left(E_{0}\right)$ (or $\left(q_{0}, \dot{q}_{0}, \ddot{q}_{0}\right)\left(E_{0}\right)$ ) and then imagine $E$ to be changed while keeping the initial conditions constant. This would affect the time relations on the trajectories and lead to a situation where the inequality (pos) could have meaning, but for general situations $t=t(E)(3.2)$ seems untenable, and hence we exclude energy-time uncertainty for completely determined microstates (see below however). Further we cannot suggest its applicability in the operator form (ETT) $\Delta E \Delta \tau \geq \hbar / 2$ since that would clash with (ET) which has a more or less substantial foundation. Thus we argue that while (ET) may be acceptable its displacement version (EET) is not, except perhaps in the averaged form $(\mathcal{E} \mathcal{E} \mathcal{T})$.

As for computation in (3.2) for example one notes that the equations in [18, 19, 20] for example have to be put in "canonical" form as in 10] and, in computing $W_{E}$, one should only differentiate terms which under a transformation $E \rightarrow E^{\prime} \neq E$ do not correspond to a Möbius transformation of $\exp (2 i W / \hbar)$ (i.e. one only differentiates terms in which $\mathcal{W}$ is changed under a transformation $E \rightarrow E^{\prime} \neq E$ ). Regarding $1-Q_{E}$ one can use the relation $W^{\prime} W_{E}^{\prime}=m\left(1-Q_{E}\right)$ for computation. As for uncertainty however my interpretation of some remarks of Floyd suggests the following approach. First I would claim that uncertainty type inequalities are incompatible with functional relations between the quantities (e.g. $p=\partial_{q} W=p(q)$ or $t=t(E)$ via $\left.W\right)$. Thus if $W$ is completely known there is generally no room for uncertainty since e.g. $\delta t \sim W_{E E} \delta E$ or with adjustment of constants $\delta t=t-t_{0}=$ $W_{E}$ completely specifies $\delta t$. Note that one of the themes in 10 involves replacing canonical transformations between independent variables $(p, q)$ with coordinate transformations $q \rightarrow \tilde{q}$ with $p=W_{q}(q)$ depending on $q$. Now we recall that the QSHJE is third order and one needs three initial conditions $\left(q_{0}, \dot{q}_{0}, \ddot{q}_{0}\right)$ or $\left(W_{0}, W_{0}^{\prime}, W_{0}^{\prime \prime}\right)$ for example to determine a solution and fix the microstate trajectories. However the Copenhagen representation uses an insufficient set of initial conditions for microstates (and literally precludes microstate knowledge). The substitute for exact microstate knowledge is then perhaps an uncertainty principle. It would be interesting to see if the two pictures interact and one could perhaps think of uncertainty relations involving $\delta t$ and $\delta E$ as in (3.2) for example as constraints for the microstate 
initial conditions. However the microstates are always compatible with the Schrödinger equation for any initial conditions and hence lead to the same operator conclusions in Hilbert space (such as (ET) for example). In any event one can continue to use the standard quantum mechanics, knowing that a deliberate sacrifice of information has been made in not specifying the background microstates (i.e. quantum mechanics in Hilbert space is imprecise by construction, leading naturally to a probabilistic theory etc.). We refrain from any further attempts at "philosophy" here.

\section{SPIN}

We follow here [8] with references to [9, 26, 27, 28, 31] (a very incomplete list). First without discussion we write down some equations from [8] and [27] ( $\hbar$ is removed in [8] so we reinsert it à la [27]). Thus one thinks of $\psi=\operatorname{Rexp}(i S / \hbar)$ with

$$
\mathcal{L}=-\rho\left[S_{t}+\frac{1}{2 m}(\nabla S)^{2}+\frac{\hbar^{2}}{8 m}\left(\frac{\nabla \rho}{\rho}\right)^{2}+V\right]
$$

$\left(\rho=R^{2}\right)$. Thence one determines the equations for a Madelung fluid

$$
S_{t}+\frac{1}{2 m}(\nabla S)^{2}+\frac{\hbar^{2}}{4 m}\left[\frac{1}{2}\left(\frac{\nabla \rho}{\rho}\right)^{2}-\frac{\Delta \rho}{\rho}\right]+V=0 ; \rho_{t}+\nabla \cdot(\rho \nabla S / m)=0
$$

(cf. also [28]). The quantum potential is $\left(|\psi|=R=\rho^{1 / 2}\right)$

$$
\frac{\hbar^{2}}{4 m}\left[\frac{1}{2}\left(\frac{\nabla \rho}{\rho}\right)^{2}-\frac{\Delta \rho}{\rho}\right]=-\frac{\hbar^{2}}{2 m} \frac{\Delta|\psi|}{|\psi|}=Q
$$

and this arises from the single nonclassical term $\left(\hbar^{2} / 8 m\right)(\nabla \rho / \rho)^{2}$ in $\mathcal{L}$ of (4.1). The internal motion or spin is related to the Zitterbewegung idea and $\vec{v} \neq \vec{p} / m$ in this context. Now one defines

$$
\vec{v}_{B}=\frac{\nabla S}{m} ; \vec{v}_{S}=\frac{\hbar \nabla \rho}{2 m \rho}
$$

and we note that the equations are invariant under $\vec{J}=\rho \vec{v}_{B} \rightarrow \vec{J}+\nabla \times \vec{b}$. This leads to a spin vector $\vec{s}$ with current velocity (4A) $\vec{v}=\vec{v}_{B}+\vec{v}_{S} \times \vec{s}=\vec{v}_{\|}+\vec{v}_{\perp}$ and (4B) $|\vec{s}|^{2}=1$ with $\vec{v}_{S} \cdot \vec{s}=0$ and $\vec{v}_{B} \cdot\left(\vec{v}_{S} \times \vec{s}\right)=0$. One notes also that $(\mathbf{4 C}) Q=-(m / 2) \vec{v}_{S}^{2}-(\hbar / 2) \nabla \cdot \vec{v}_{S}$. For stationary states $\psi=\psi(q) \exp (-i E t / \hbar)$ with $\psi(q)=\operatorname{Rexp}(i W / \hbar)$ one has then

$$
\frac{1}{2 m}(\nabla W)^{2}-\frac{\hbar^{2}}{2 m} \frac{\Delta R}{R}+V-E=0 ; \nabla \cdot(\rho \nabla W)=0
$$

with $Q$ given in (4.3).

We want to see now if we can relate the spin picture to the trajectory representation. Since we are dealing with the same basic Schrödinger equation the only new feature is 3-D. 
One can speak of internal motion, spin, Zitterbewegung, etc. but once a current velocity $\vec{v}$ appears as in (4A) we are at least implicitly making contact with the idea of particle motion and some comparison between $\vec{v}$ and trajectory velocity $d \vec{q} / d t$ should be possible and meaningful. This point may be arguable but we assume it momentarily at least. Actually in [8] one explicitly deals with $\vec{v}$ as a particle velocity so this should carry over to the stationary state. However the arguments in [8] about trajectory representations do not take into account the work of FM or Floyd involving microstates so we feel the conclusions in [8] should be correspondingly adjusted (see below for more on this). We will show that the use of current velocity $\vec{v}$ as particle velocity seems to be incorrect.

Thus following the 1-D example of (2.4) where $m\left(1-Q_{E}\right) \dot{q}=W^{\prime}$ we use the relation $t \sim \partial_{E} W$ again to suggest $(\vec{x} \sim \vec{q})$

$$
t-t_{0}=\partial_{E} \int_{\vec{x}_{0}}^{\vec{x}} \nabla W \cdot d \vec{x} \sim \nabla t=\nabla W
$$

Then from (4.5) one has

$$
\frac{1}{n} \nabla W \cdot \nabla W_{E}+Q_{E}-1=0 ; \nabla \cdot\left[\partial_{E}(\rho \nabla W)\right]=0
$$

We can write then $(\mathbf{4 D}) \partial_{E}(\rho \nabla W)=\nabla \times \vec{\gamma}$ say and following $d t / d q=\partial_{E} W^{\prime}=W_{E}^{\prime}$ in $1-\mathrm{D}$, or $d q / d t=1 / W_{E}^{\prime}$, we suggest for (4.6) the relation (4E) $\partial t / \partial x_{i}=\partial W_{E} / \partial x_{i} \sim \dot{x}_{i}=$ $1 / \partial_{i} W_{E}$. In a similar manner (4.7) leads to

$$
\nabla W \cdot \nabla t=m\left(1-Q_{E}\right) ; m \dot{x}_{i}=\frac{|\nabla W|^{2}}{\left(1-Q_{E}\right) \partial_{i} W}
$$

which implies $(\mathbf{4 F}) m\left(1-Q_{E}\right)(d \vec{x} / d t) \cdot \nabla W=3|\nabla W|^{2}$.

Now the constructions of $\|8\|$ with $\vec{v}_{B} \cdot\left(\vec{v}_{S} \times \vec{s}\right)=0$ as in (4B) give $\vec{v} \cdot \vec{v}_{B}=\left|\vec{v}_{B}\right|^{2}$ where $\vec{v}_{B}=(1 / m) \nabla W$. If one could identify $\vec{v}$ with $d \vec{x} / d t$ there would result then from (4F) the formula $(\mathbf{4 G})\left(1-Q_{E}\right)=3 \mathrm{~m}$ which is unlikely at best. Hence we conclude that the current velocity $\vec{v}$ of [8] cannot be identified with particle velocity and the conclusions there about trajectories are not correct. Perhaps the difficulty lies in the following observation. Even though the quantum potential $Q$ can be recovered from $\vec{v}_{S}$ as in (4C) nevertheless the full quantum potential is not used in constructing $\vec{v}_{S}$ via (4.4) as can be seen from (4.3). In the trajectory picture from FM or Floyd the full quantum potential is used in determining $d \vec{x} / d t$ as indicated in 4.8 ) or $(\mathbf{4 F})$.

A few additional comments should also be made about adapting the development in [8] or 27 for stationary state situations. Thus from (4.5) we first extend $\vec{J}$ in the form $\vec{J}=\rho \vec{v}_{B} \rightarrow \rho \vec{v}_{B}+\nabla \times(\rho \vec{c})$ and satisfy the equation $\nabla \cdot \vec{J}=0$ via $(\mathbf{4 H}) \vec{J}=\rho \vec{v}_{B}+\nabla \times(\rho \vec{c})=$ $\nabla \times \vec{\phi}$ with $\vec{s}=(2 m / \hbar) \vec{c}$ and $\vec{\phi}$ dependent on $\vec{x}$. Then (cf. (4.4) $) \nabla \cdot \vec{J}=0 \sim \nabla \cdot\left(\rho \vec{v}_{B}\right)=0$ and one can write

$$
\nabla \times(\rho \vec{c})=\nabla \rho \times \vec{c}+\rho(\nabla \times \vec{c})=\rho\left[\vec{v}_{S} \times \vec{s}+\frac{\hbar}{2 m} \nabla \times \vec{s}\right]
$$


with

$$
\vec{v}=\vec{v}_{B}+\vec{v}_{S} \times \vec{s} ; \quad \vec{J}=\rho \vec{v}+\vec{J}_{0}=\nabla \times \vec{\phi}=\vec{\eta} ; \quad \overrightarrow{J_{0}}=\frac{\hbar \rho}{2 m} \nabla \times \vec{s}
$$

One can think of $\overrightarrow{J_{0}}$ as a "pseudocurrent" added on to deal with cases of a variable spin vector $\vec{s}(\vec{x})$ and $\vec{s}$ still is to satisfy (4B). The term $\vec{\phi}$ is added here to give a variable right side for $\vec{J}$. Now in order to determine if this produces a solvable configuration we note that the equations (4B) consist of three equations in three unknowns $s_{i}$ for $\vec{s}=\left(s_{1}, s_{2}, s_{3}\right)$ and the coefficients in these equations depend on $\nabla W$ via $\vec{v}_{B}$ and on $\rho$ via $\vec{v}_{S}$ yielding $\vec{s}=\vec{s}(\rho, \nabla W)$. We think of $\vec{\eta}$ as arbitrary for the moment and from $(\mathbf{4} \mathbf{J}) \vec{v}=(1 / \rho)\left[\vec{\eta}-\overrightarrow{J_{0}}\right]$ one obtains via (4.2) and (4.5)

$$
|\vec{v}|^{2}=\left|\vec{v}_{B}\right|^{2}+\left|\vec{v}_{S}\right|^{2}=\frac{2}{m}(E-V)+\frac{\hbar^{2}}{2 m^{2}} \frac{\Delta \rho}{\rho}=\left|\frac{\vec{\eta}}{\rho}-\frac{\hbar}{2 m} \nabla \times \vec{s}\right|^{2}
$$

Thus we have two more equations, namely (4.10) and (4J) in the form $(\mathbf{4 K}) \vec{v}=(1 / m) \nabla W+$ $(\hbar / 2 m)(\nabla \rho / \rho)=(\vec{\eta} / \rho)-(\hbar / 2 m) \nabla \times \vec{s}$. If we put $\vec{s}=\vec{s}(\rho, \nabla W)$ in these equations they become two equations (4.10) and (4K) for $\rho$ and $\nabla W$ in terms of an arbitrary $\vec{\eta}$. Thus in principle this configuration should be solvable and some preliminary calculations are promising. As an example take $\vec{s}=\vec{i}$ and $\nabla \rho=\rho_{2} \vec{j}$ so curl $\vec{s}=0$ and $\nabla \rho \times \vec{s}=-\rho_{2} \vec{k}$. Then $\vec{v}_{B} \perp(\nabla \rho \times \vec{s})$ will imply $\nabla W=W_{1} \vec{i}+W_{2} \vec{j}$ and one has $(\mathbf{4 L}) \vec{\eta} / \rho=(1 / m)\left(W_{1} \vec{i}+W_{2} \vec{j}\right)-(\hbar \rho / 2 m \rho) \vec{k}$. Thus given $\vec{\eta}$ we must have

$$
\frac{\rho W_{1}}{m}=\eta_{1} ; \frac{\rho W_{2}}{m}=\eta_{2} ;-\frac{\hbar \rho_{2}}{2 m}=\eta_{3}
$$

along with (4.10) in the form

$$
\frac{2 \rho^{2}}{m}(E-V)+\frac{\hbar^{2} \rho \rho_{22}}{2 m^{2}}=\frac{\rho^{2}}{m^{2}}\left(W_{1}^{2}+W_{2}^{2}\right)+\frac{\hbar^{2} \rho_{2}^{2}}{4 m^{2}}
$$

This can be satisfied if e.g. $(\mathbf{4 M}) 2(E-V)=W_{1}^{2}+W_{2}^{2}$ and $\rho \rho_{22}=(1 / 2) \rho_{2}^{2}$. The latter equation is $\rho_{22} / \rho_{2}=(1 / 2)\left(\rho_{2} / \rho\right)$ leading to $\log \left(\rho_{2} / \rho^{1 / 2}\right)=f(x, z)$ and eventually $2 \rho^{1 / 2}=$ $\alpha(x, z) y+\beta(x, z)$ with $\alpha, \beta$ constant. Evidently $(4 \mathbf{M})$ requires also $V=V(x, y)$.

ACKNOWLEDGEMENT The author would like to thank E. Floyd and M. Matone for valuable comments. 


\section{References}

[1] Y. Aharonov and D. Bohm, Phys. Rev., 122/5 (1961), 1649-1658; 134/6B (1964), 14171418

[2] D. Bohm and B. Hiley, The undivided universe, Routledge, London, 1993

[3] D. Bohm, Phys. Rev., 85 (1952), 166-179 and 180-193

[4] J. Briggs and J. Rost, quant-ph 9902035

[5] J. Cushing, Quantum mechanics, Univ. Chicago Press, 1994

[6] V. Delgado and J. Muga, Phys. Rev. A, 56 (1997), 3425-3435

[7] J. Eberly and L. Singh, Phys. Rev. D, 7 (1973), 359-362

[8] S. Esposito, quant-ph 9902019

[9] S. Esposito, Found. Phys., 28 (1998), 231-244; Phys. Lett. A, 225 (1997),203-209

[10] A. Faraggi and M. Matone, hep-th 9809127

[11] A. Faraggi and M. Matone, Phys. Rev. Lett., 78 (1997), 163-166

[12] A. Faraggi and M. Matone, hep-th 9705108 = Phys. Lett. B, 450 (1999), 34; hep-th $9711028=$ Phys. Lett. B, 437 (1998), 369; hep-th $9801033=$ Phys. Lett. A, 249 (1998), 180; hep-th $9809125=$ Phys. Lett. B, 445 (1999), 77; and hep-th 9809126= Phys. Lett. B, 445 (1999), 357

[13] B. Felsager, Geometry, particles, and fields, Springer, 1998

[14] E. Fick and F. Engelmann, Zeits. für Physik, 175 (1963), 271-282; 178 (1964), 551-562

[15] E. Floyd, Phys. Rev. D, 26 (1982), 1339-1347; Phys. Rev. D, 29 (1984), 1842-1844; Phys. Rev. D, 25 (1982), 1547-1551

[16] E. Floyd, Phys. Lett. A, 214 (1996), 259-263

[17] E. Floyd, Phys. Rev. D, 34 (1986), 3246-3249

[18] E. Floyd quant-ph 9707051; quant-ph 9708007; quant-ph 9708026

[19] E. Floyd, Phys. Essays, 7 (1994), 135-145

[20] E. Floyd, Ann. Fond. L. deBroglie, 20 (1995), 263-279

[21] B. Hiley and F. Peat, Quantum implications, Routledge, London, 1987

[22] P. Holland, The quantum theory of motion, Cambridge Univ. Press, 1997 
[23] M. Morrison, Understanding quantum mechanics, Prentice-Hall, 1990

[24] E. Papp, The uncertainty principle and foundations of QM, Wiley, 1977, pp. 29-50

[25] D. Park, Classical dynamics and its quantum analogues, Springer, 1990

[26] M. Razavy, Nuovo Cimento, 63 (1969), 271-308; Amer. Jour. Phys., 35 (1967),955-960

[27] E. Recami and G. Salesi, Phys. Rev. D, 57 (1998), 98-105

[28] G. Salesi, Mod. Phys. Lett. A, 11 (1996), 1815-1823

[29] G. Salesi and E. Recami, Phys. Lett. A, 190 (1994), 137-143

[30] F. Schwabl, Quantum mechanics, Springer, 1995

[31] T. Takabayashi, Prog. Theor. Phys., 69 (1983), 1323-1344 